\title{
Application of advanced optical methods for classification of air contaminants
}

\author{
M. Wlodarski, K. Kopczyński, M. Kaliszewski, M. Kwaśny, \\ M. Mularczyk-Oliwa \& M. Kastek \\ Institute of Optoelectronics, Military University of Technology, \\ Warsaw, Poland
}

\begin{abstract}
Biochemical, highly sensitive methods of pollutant identification have common drawbacks - relatively long analysis time and the necessity of on-site sample collection. Optical methods are less sensitive and less selective, but they allow real time analysis. These optical methods include Fourier Transform Infrared Spectroscopy (FTIR), and Laser Induced Fluorescence (LIF). Both methods give the possibility of stand-off detection. The aim of the presented work is to study the possibilities of detection and classification of air contaminants, based on their optical properties. In this work we present measurement results of UV-VIS fluorescence characteristics and IR optical absorption of vegetable pollens, bacterial spores, and non-biological air contaminants (diesel fuel, dust, syloid). Results of the principal components analysis show that both LIF and FTIR methods allow distinguishing between groups of materials, i.e. pollens and nonbiological materials, thus making application of these methods for detection and preliminary classification possible.
\end{abstract}

Keywords: biological contaminants, LIF, FTIR, stand-off detection, UV-VIS fluorescence, pollution monitoring.

\section{Introduction}

Air pollutants have strong impacts on human health. Inhaled particulate matter is often responsible for severe side effects like respiratory tract infections caused by bacterial or viral agents and allergic reactions triggered by pollens.

Modern biochemical assays, although highly sensitive and specific, are time consuming. The fast analysis is important in case of potential warfare attack (Pan 
et al [1]). For this reason, methods based on optical or spectral properties of contaminants, for detection and analysis have become more important in the last decade (Hairston et al [2], Pan et al [3]).

In our project we are currently developing the application of two optical methods of biological pollution monitoring: passive FTIR spectroscopy, and dual wavelength fluorescence LIDAR for fast stand-off detection and classification.

Biological materials excited with UV light show strong fluorescence in two bands. The first, more intensive band has excitation maximum at about $280 \mathrm{~nm}$, and emission maximum at about $330 \mathrm{~nm}$, and comes mainly from fluorescence of Tryptophan. Second band is result of NADH fluorescence, with excitation and emission maxima at about $340 \mathrm{~nm}$ and at about $450 \mathrm{~nm}$ respectively (Wlodarski et al [4]).

\section{Materials and methods}

\subsection{Excitation-emission matrices}

Fluorescence spectra of the samples were measured with FS 900 spectrofluorimeter (Edinburgh Instruments) provided with a front-surface holder that eliminates inner filter effect. A sample solution was placed in $1 \mathrm{~cm}$ length quartz cuvette. The solutions were mixed during the experiment with a magnetic stirrer. The excitation wavelength ranged from 250 to $400 \mathrm{~nm}$ in $5 \mathrm{~nm}$ increments, and emission was scanned from 260 to $550 \mathrm{~nm}$ with step $2 \mathrm{~nm}$. The slits on both monochromators were set for 2-nm bandwidth. The Raman signal and the background were subtracted from the sample spectra.

Table 1: $\quad$ Alphabetical list of measured materials.

\begin{tabular}{|l|l|l|}
\hline Sample name & Group & Abbrev. \\
\hline AC Fine Test Dust & Non-biological & DUST \\
\hline Bacillus atrophaeus spores (Edgewood) & Bacterial spores & BGst \\
\hline Bacillus thuringiensis spores (Turex) & Bacterial spores & BTst \\
\hline Bermuda grass pollen & Vegetable pollens & BER \\
\hline Bermuda grass smut spores & Vegetable smut spores & BERs \\
\hline Black walnut pollen & Vegetable pollens & WAL \\
\hline Corn pollen & Vegetable pollens & COR \\
\hline Diesel fuel & Non-biological & OIL \\
\hline Johnsons grass smut spores & Vegetable smut spores & JON \\
\hline Paper mulberry pollen & Vegetable pollens & PAP \\
\hline Pecan pollen & Vegetable pollens & PEC \\
\hline Ragweed pollen & Vegetable pollens & RAG \\
\hline Syloid & Non biological & SYL \\
\hline
\end{tabular}




\subsubsection{Chemicals}

Technical preparation of Bacillus turingiensis spores (Turex) and Bacillus atrophaeus spores were gently provided from PvTT, Tampere, Finland. Pollens and AC Fine Test Dust standard were obtained from Duke Scientific.Corp, Faber Place, Palo Alto, CA. L-Tryptophan was obtained from Sima-Aldrich, Poland. Syloid 244 FP was from Grace GmbH\&Co. KG. Diesel fuel was commercially available from petrol station. All chemicals were of the highest purity commercially available.

\subsubsection{Stock solutions}

Solutions were prepared from powders. Distillated water was added to obtain the final concentration $1 \mathrm{mg} / \mathrm{ml}$. In some cases it was necessary to dilute prepared samples due to a strong fluorescence signal that caused detector saturation.

\subsection{Laser Induced Fluorescence}

LIF spectra were measured with the ICDD camera (Princeton Instruments PI-MAX 2) connected to monochromator (Acton Research Corporation SpectraPro 2150i). The exposure time of the camera was set to $100 \mathrm{~ms}$. High signal-to-noise ratio, was obtained by averaging 100 single measurements of spectrum. The spectrometer resolution was set to $5 \mathrm{~nm}$. Two lasers were used for excitation: $266 \mathrm{~nm}$ (Intelite Inc. quadrupled Nd:YAG, $20 \mathrm{~mW}$ average power), and $375 \mathrm{~nm}$ (Power Technology Inc. laser diode, $15 \mathrm{~mW}$ power). Laser wavelengths were chosen in consideration with fluorescence LIDAR requirements, therefore they are not the same as optimal excitation wavelengths for biological materials. Lasers and monochromator were connected to a measurement chamber via optical fibers. Laser signal was rejected from emission spectra using two Semrock long-pass filters: FF-300LP, and FF-409LP for $266 \mathrm{~nm}$ and $375 \mathrm{~nm}$ excitation respectively. Fluorescence was measured in the range of $300-550 \mathrm{~nm}$ for $266 \mathrm{~nm}$ excitation, and $390-640 \mathrm{~nm}$ for $375 \mathrm{~nm}$ excitation. Solid samples were fixed between two thin quartz plates. The diesel fuel sample was measured in quartz $1 \mathrm{~cm}$ cuvette. Fluorescence signal was collected from the surface of the sample to avoid inner filter effect.

\subsection{Medium infrared absorption}

IR absorption spectra were collected using Perkin-Elmer Spectrum GX Optica FTIR spectrometer. Measurement range was $4000 \mathrm{~cm}^{-1}-650 \mathrm{~cm}^{-1}(2.5 \mu \mathrm{m}-$ $12.5 \mu \mathrm{m}$ ) with $4 \mathrm{~cm}^{-1}$ resolution. Two methods of measurement were applied: Diffuse Reflectance Infrared Fourier Transform (DRIFT), and Horizontal Attenuated Total Reflectance (HATR).

DRIFT technique allows for measurement of solid, highly absorbing samples ([5], Brimmer et al [6]). HATR technique allows for direct measurement of liquid samples [7].

Solid samples for DRIFT measurements were mixed with $\mathrm{KBr}$ powder in $5 \%$ proportion. Diesel fuel was measured in liquid form using the HATR technique. 
Both techniques allow measurements from a small amount of samples. Those methods were chosen as promising for real-time monitoring applications.

\subsection{Principal Components Analysis}

PCA was conducted using SIMCA-P 11.5 program from Umetrics AB. Results were presented in the form of score plots.

\section{Results and discussion}

\subsection{Excitation-emission matrices}

Fluorescence spectra recorded in map mode provide full information on excitation and emission maxima. Presented matrices are normalized to the maximum of emission intensity.

Representative EX-EM maps are shown in Figure 1. The shapes and intensities vary between different materials.
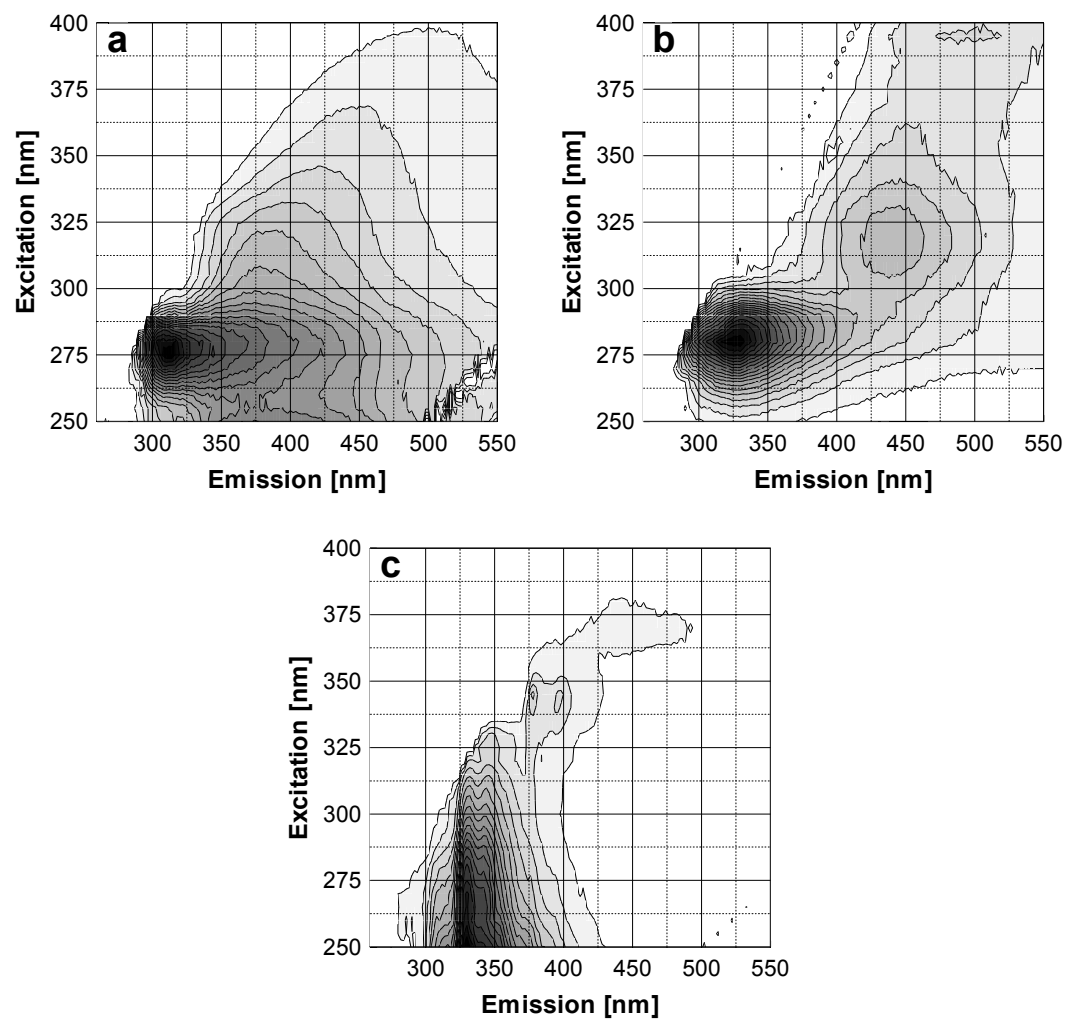

Figure 1: $\quad$ EX-EM matrices of: a) Turex, b) Paper Mulberry Pollen, c) Diesel fuel. 


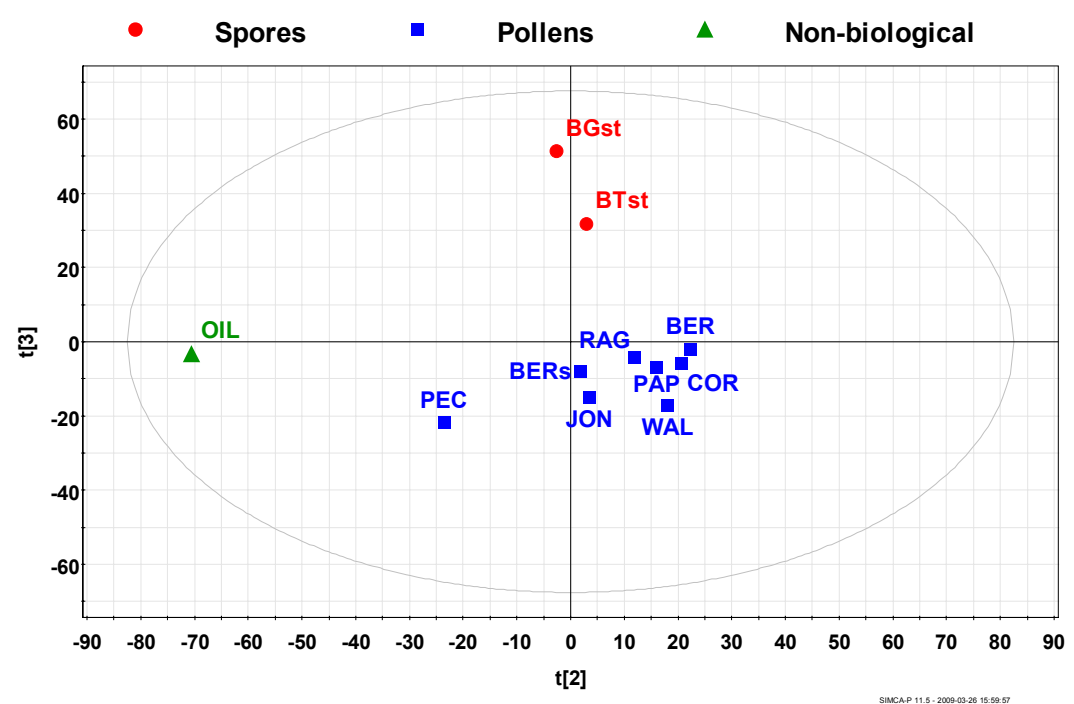

Figure 2: $\quad$ PCA results of Excitation-Emission matrices.

Classification with PCA method shows that particular groups are well separated (Figure 2). Syloid and dust were not analyzed due to lack of fluorescence. Oil, as a non-biological material, is placed in a distant position compared to spores and pollens.

\subsection{Laser Induced Fluorescence spectra}

Laser Induced Fluorescence is promising technique for stand-off detection of biological contamination. Analysis of EX-EM matrices allows for optimal choice

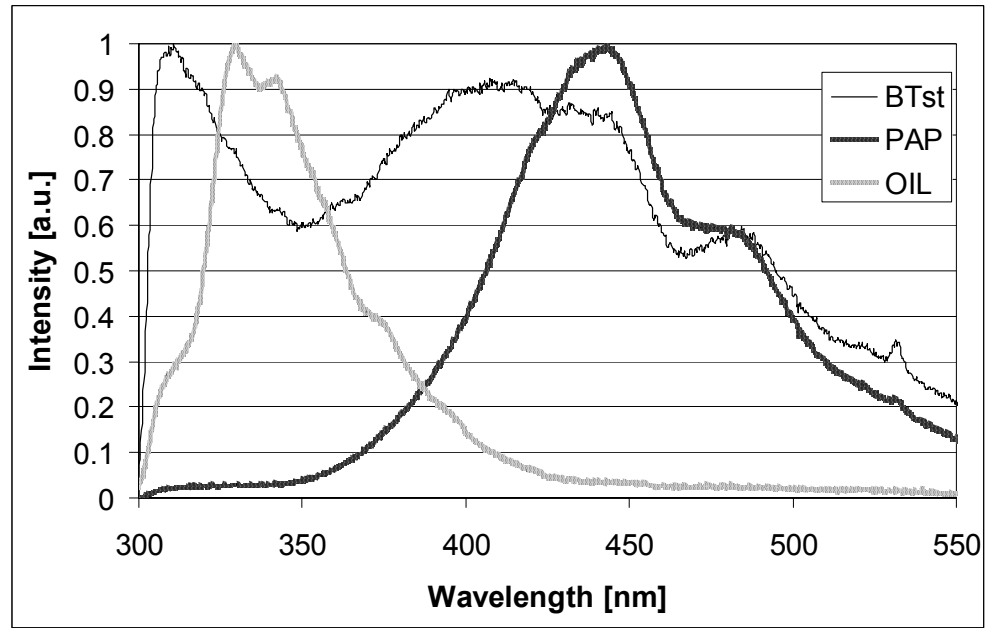

Figure 3: $\quad$ Selected LIF spectra, excitation $266 \mathrm{~nm}$. 


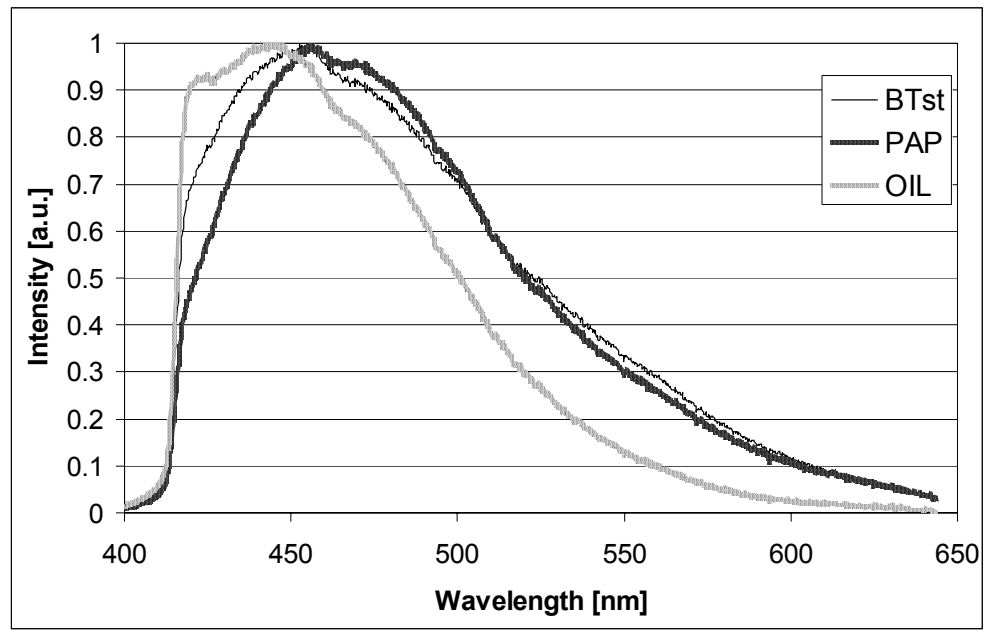

Figure 4: $\quad$ Selected LIF spectra, excitation $375 \mathrm{~nm}$.

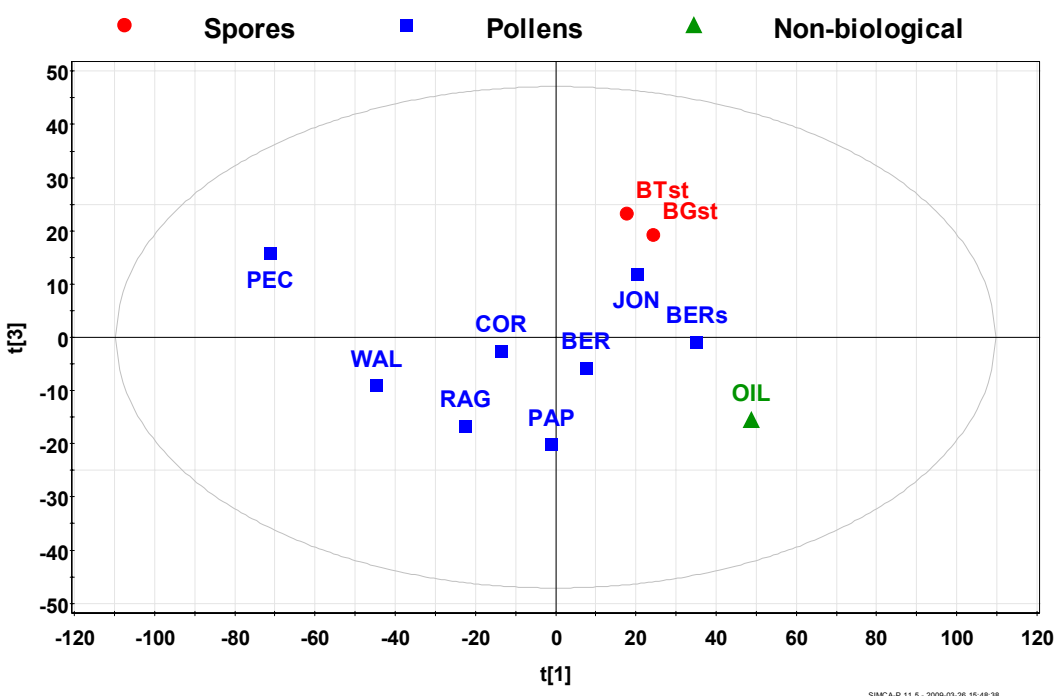

Figure 5: $\quad$ PCA results of LIF spectra.

of light source. LIF spectra recorded at excitation $266 \mathrm{~nm}$ differ significantly (Figure 3), while those from excitation at $375 \mathrm{~nm}$ are very similar (Figure 4).

PCA of LIF spectra presented in Figure 5 show that bacterial spores are forming separate group. Discrimination of pollens is unequivocal because of considerable distribution over the plot area.

PCA graphs of whole EX-EM maps (Figure 2) show more precise discrimination comparing to LIF (Figure 5). Differences can be explained by 


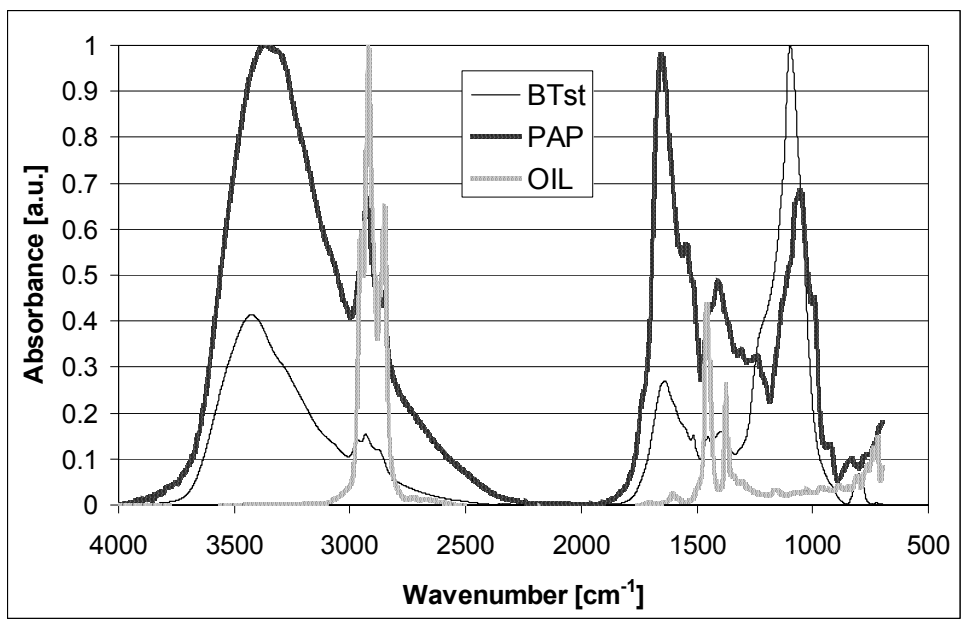

Figure 6: $\quad$ Selected FTIR spectra.

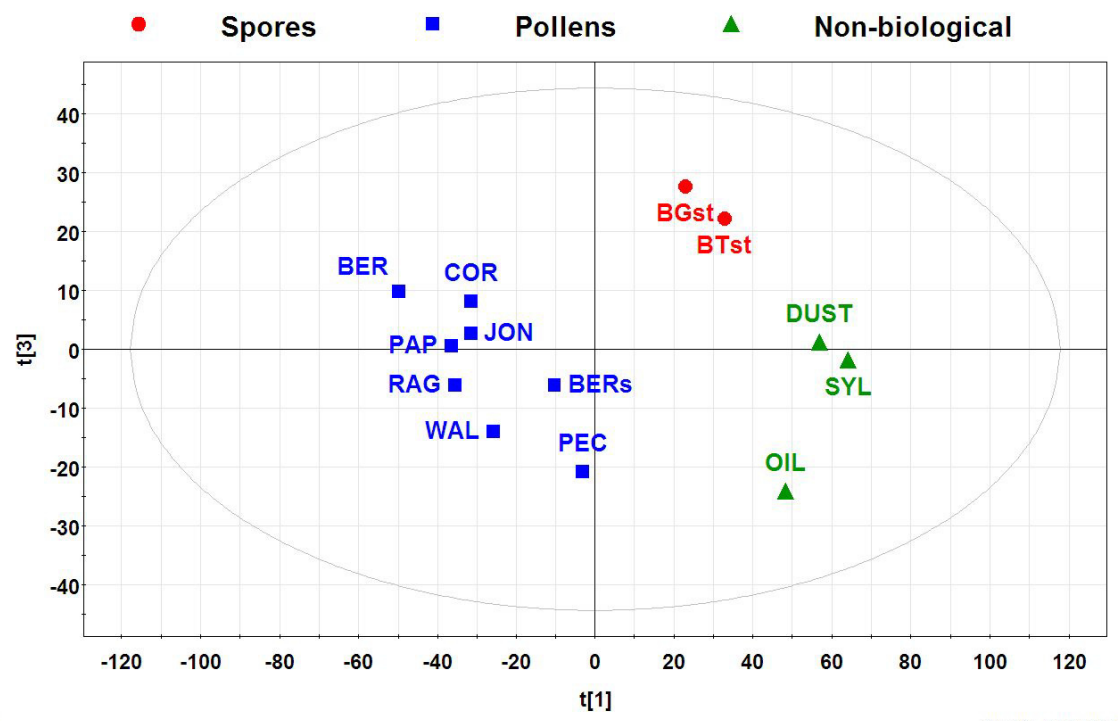

Figure 7: $\quad$ PCA results of FTIR spectra.

30 excitation wavelengths included in map while LIF applies only two. Despite reduction of data it is still possible to differentiate between biological and nonbiological material.

\subsection{Medium Infrared absorption spectra}

Infrared absorption spectroscopy has been widely used for identification of specific groups and chemical bondings. The great advantage of this method is identification of non-fluorescent substances. 
Despite very complex composition, biological substances contain similar groups of proteins, lipids, organic and inorganic elements. Their presence is manifested by absorption peaks at specific region of the spectrum.

Normalized spectra of three different substances are presented in Figure 6. The plots exhibit considerable differences mainly due to intensities of the absorption peaks. That feature is common for biological samples like PAP and BTst. Generally, Diesel fuel absorbs IR light in different spectral regions than biological samples. The only similarity is apparent in region $3000-2800 \mathrm{~cm}^{-1}$ that is characteristic to $\mathrm{C}-\mathrm{H}$ bounds.

Results of PCA analysis of IR spectra are shown in Figure 7. Points representing particular groups are well separated from each other.

\section{Conclusions}

Spectroscopy in infrared region showed better discrimination than fluorescence methods. IR spectra provide more information due to higher number of different peaks. Simultaneous application of passive FTIR and fluorescence LIDAR provides better independence from environmental conditions during monitoring (i.e. FTIR for daytime and LIDAR for nighttime application). Identification of biological air pollutants with advanced optical systems can be applied for both civil and military purposes. Unquestionable advantages are continuous, realtime, and stand-off monitoring. Today's FTIR and LIDAR technologies allow stand-off detection of chemical aerosol contaminants (Mierczyk et al [8], Killinger [9]). Creation of biological material database is unavoidable and very important element for development of monitoring systems. Our results show that preliminary classification of biological air contaminants using advanced optical methods is possible.

\section{References}

[1] Y-L. Pan, V. Boutou, J. Bottiger, S. Zhang, J-P. Wolf, R. Chang. A Puff of Air Sorts Bioaerosols for Pathogen Identification, Aeros. Sci. and Techn, 38, 2004, 598-602.

[2] P.P. Hairston, J. Ho, F.R. Quant. Design of an instrument for real-time detection of bioaerosols using simultaneous measurement of particle aerodynamic size and intrinsic fluorescence, J. Aerosol Sci., 28, 3, 1997, 471-482.

[3] Y-L Pan, J. Hartings, R.G. Pinnick, S.C. Hill, J. Halverson, R.K. Chang. Single-Particle fluorescence spectrometer for ambient aerosols, Aerosol Sci. Technol., 37, 8, 2003, 627-638.

[4] M. Wlodarski, M. Kaliszewski, M. Kwasny, Z. Zawadzki, K. Kopczynski Z. Mierczyk, J. Mlynczak E. Trafny, and M. Szpakowska, Fluorescence excitation-emission maps database of biological agents, Proc. SPIE, 6398, 06-1-12 (2006).

[5] Diffuse Reflectance Accessory; User's Guide, Copyright (C) 1998 The Perkin-Elmer Corporation United Kingdom 
[6] Paul J. Brimmer, Peter R. Griffiths, and N. J. Harrick, Angular Dependence of Diffuse Reflectance Infrared Spectra. Part I: FT-IR Spectrogoniophotometer, Appl. Spectrosc. 40, 258-265 (1986)

[7] Horizontal ATR Accessory; User's Guide, Copyright (C) 1998 The PerkinElmer Corporation United Kingdom

[8] Z. Mierczyk, M. Zygmunt, A. Gawlikowski, A. Gietka, M. Kaszczuk, P. Knysak, A. Młodzianko, M. Muzal, W. Piotrowski, J. Wojtanowski, Twowavelength backscattering lidar for stand-off detection of aerosols, Proceedings of SPIE, Vol. 7III, 7III0R, SPIE Europe Remote Sensing, Cardiff (Wales), (2008).

[9] Dennis Killinger, Detecting chemical, biological, and explosive agents, SPIE Newsroom, archived invited paper, August 2006. 